\title{
Formation of Naked Singularities: The Violation of Cosmic Censorship
}

\author{
Stuart L. Shapiro and Saul A. Teukolsky \\ Center for Radiophysics and Space Research and Departments of Astronomy and Physics, \\ Cornell University, Ithaca, New York 14853
}

(Received 7 September 1990)

\begin{abstract}
We use a new numerical code to evolve collisionless gas spheroids in full general relativity. In all cases the spheroids collapse to singularities. When the spheroids are sufficiently compact, the singularities are hidden inside black holes. However, when the spheroids are sufficiently large, there are no apparent horizons. These results lend support to the hoop conjecture and appear to demonstrate that naked singularities can form in asymptotically flat spacetimes.
\end{abstract}

PACS numbers: $04.20 . \mathrm{Jb}, 95.30 . \mathrm{Sf}$, 97.60.Lf

It is well known that general relativity admits solutions with singularities, and that such solutions can be produced by the gravitational collapse of nonsingular, asymptotically flat initial data. The cosmic censorship hypothesis ${ }^{1}$ states that such singularities will always be clothed by event horizons and hence can never be visible from the outside (no naked singularities). If cosmic censorship holds, then there is no problem with predicting the future evolution outside the event horizon. If it does not hold, then the formation of a naked singularity during collapse would be a disaster for general relativity theory. In this situation, one cannot say anything precise about the future evolution of any region of space containing the singularity since new information could emerge from it in a completely arbitrary way.

Are there guarantees that an event horizon will always hide a naked singularity? No definitive theorems exist. Counterexamples ${ }^{2}$ are all restricted to spherical symmetry and typically involve shell crossing, shell focusing, or self-similarity. Are these singularities an accident of spherical symmetry?

For nonspherical collapse Thorne $^{3}$ has proposed the hoop conjecture: Black holes with horizons form when and only when a mass $M$ gets compacted into a region whose circumference in every direction is $\mathcal{C} \lesssim 4 \pi M$. If the hoop conjecture is correct, aspherical collapse with one or two dimensions appreciably larger than the others might then lead to naked singularities.

For example, consider the Lin-Mestel-Shu instability ${ }^{4}$ for the collapse of a nonrotating, homogeneous spheroid of collisionless matter in Newtonian gravity. Such a configuration remains homogeneous and spheroidal during collapse. If the spheroid is slightly oblate, the configuration collapses to a "pancake," while if the spheroid is slightly prolate, it collapses to a spindle. While in both cases the density becomes infinite, the formation of a spindle during prolate collapse is particularly worrisome. The gravitational potential, gravitational force, tidal force, and kinetic and potential energies all blow up. This behavior is far more serious than mere shell crossing, where the density alone becomes momentarily infi- nite. For collisionless matter, prolate evolution is forced to terminate at the singular spindle state. For oblate evolution, the matter simply passes through the pancake state, but then becomes prolate and also evolves to a spindle singularity.

Does this Newtonian example have any relevance to general relativity? We already know that infinite cylinders do collapse to singularities in general relativity, and, in accord with the hoop conjecture, are not hidden by event horizons. ${ }^{3,5}$ But what about finite configurations in asymptotically flat spacetimes?

Previously, we constructed ${ }^{6}$ an analytic sequence of momentarily static, prolate, and oblate collisionless spheroids in full general relativity. We found that in the limit of large eccentricity the solutions all become singular. In agreement with the hoop conjecture, extended spheroids have no apparent horizons. Can these singularities arise from the collapse of nonsingular initial data? To answer this, we have performed fully relativistic dynamical calculations of the collapse of these spheroids, starting from nonsingular initial configurations.

We find that the collapse of a prolate spheroid with sufficiently large semimajor axis leads to a spindle singularity without an apparent horizon. Our numerical computations suggest that the hoop conjecture is valid, but that cosmic censorship does not hold because a naked singularity may form in nonspherical relativistic collapse.

Numerical code.-Our numerical code solves Einstein's equations for the evolution of nonrotating, collisionless matter in axisymmetric spacetimes. The field equations are expressed in $3+1$ form following Arnowitt, Deser, and Misner. ${ }^{7}$ We use maximal time slicing and isotropic spatial coordinates. The field equations ${ }^{8}$ reduce to a coupled set of nonlinear hyperbolic and elliptic partial differential equations which we solve by finite differencing. The matter equations are the geodesic equations in the self-consistent gravitational field. The numerical treatment is a mean-field particle simulation scheme that solves the Vlasov equation in general relativity. It is an extension of our previous relativistic treat- 
ment for spherical spacetimes ${ }^{9}$ and our Newtonian method ${ }^{10}$ for axisymmetric configurations. The code can handle matter velocities approaching the speed of light and strong gravitational fields, including black holes. It is designed to treat cases in which the collisionless matter collapses to a singularity: Specifically, oblate collapse to flat pancakes and prolate collapse to thin spindles.

We have carried out a large battery of test-bed calculations to ensure the reliability of the code. These tests included the propagation of linearized analytic quadrupole waves with and without matter sources and nonlinear Brill waves in vacuum spacetimes; maintaining equilibria and identifying the onset of instability for

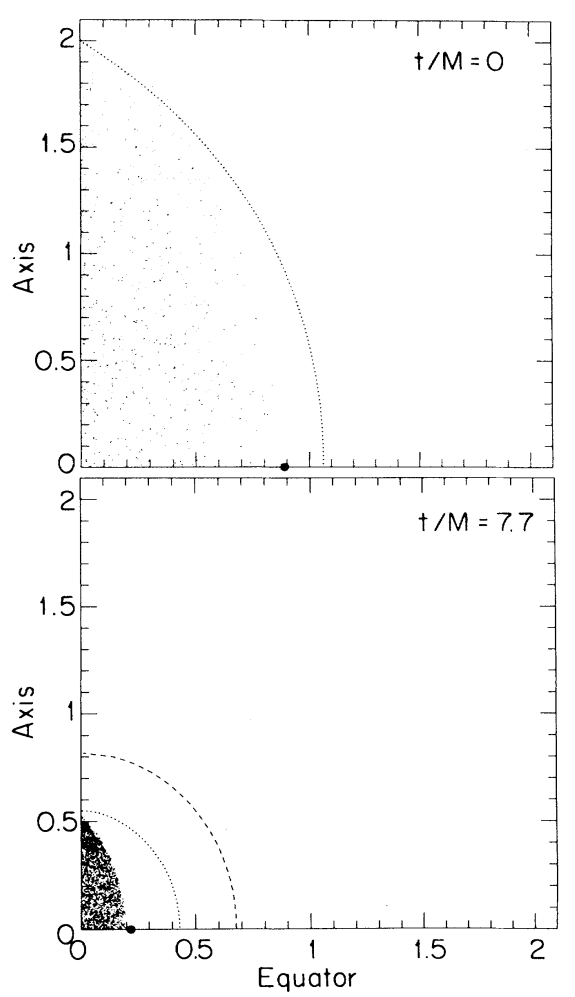

FIG. 1. Snapshots of the particle positions at initial and late times for prolate collapse. The positions (in units of $M$ ) are projected onto a meridional plane. Initially the semimajor axis of the spheroid is $2 M$ and the eccentricity is 0.9 . The collapse proceeds nonhomologously and terminates with the formation of a spindle singularity on the axis. However, an apparent horizon (dashed line) forms to cover the singularity. At $t / M=7.7$ its area is $\mathcal{A} / 16 \pi M^{2}=0.98$, close to the asymptotic theoretical limit of 1. Its polar and equatorial circumferences at that time are $\mathcal{C}_{\mathrm{pole}}^{\mathrm{AH}} / 4 \pi M=1.03$ and $\mathcal{C}_{\mathrm{eq}}^{\mathrm{AH}} / 4 \pi M=0.91$. At later times these circumferences become equal and approach the expected theoretical value 1 . The minimum exterior polar circumference is shown by a dotted line when it does not coincide with the matter surface. Likewise, the minimum equatorial circumference, which is a circle, is indicated by a solid dot. Here $\mathcal{C}_{\text {eq }}^{\min } / 4 \pi M=0.59$ and $\mathcal{C}_{\text {pole }}^{\min } / 4 \pi M=0.99$. The formation of a black hole is thus consistent with the hoop conjecture. spherical equilibrium clusters; ${ }^{9}$ reproducing Oppenheimer-Snyder collapse of homogeneous dust spheres and Newtonian collapse of homogeneous spheroids. ${ }^{4,10} \mathrm{We}$ constructed a number of geometric probes to diagnose the evolving spacetime. We tracked the Brill mass and outgoing radiation energy flux to monitor mass-energy conservation. To confirm the formation of a black hole, we probed the spacetime for the appearance of an apparent horizon and computed its area and shape when it was present. To measure the growth of a singularity, we computed the Riemann invariant $I \equiv R_{\alpha \beta \gamma \delta} R^{\alpha \beta \gamma \delta}$ at every spatial grid point. To test the hoop conjecture, we computed the minimum equatorial and polar circumferences outside the matter.

Typical simulations were performed with a spatial grid of 100 radial and 32 angular zones, and with 6000 test particles. A key feature enabling us to snuggle close to singularities was that the angular grid could fan and the radial grid could contract to follow the matter.

Collapse of collisionless spheroids. - We followed the

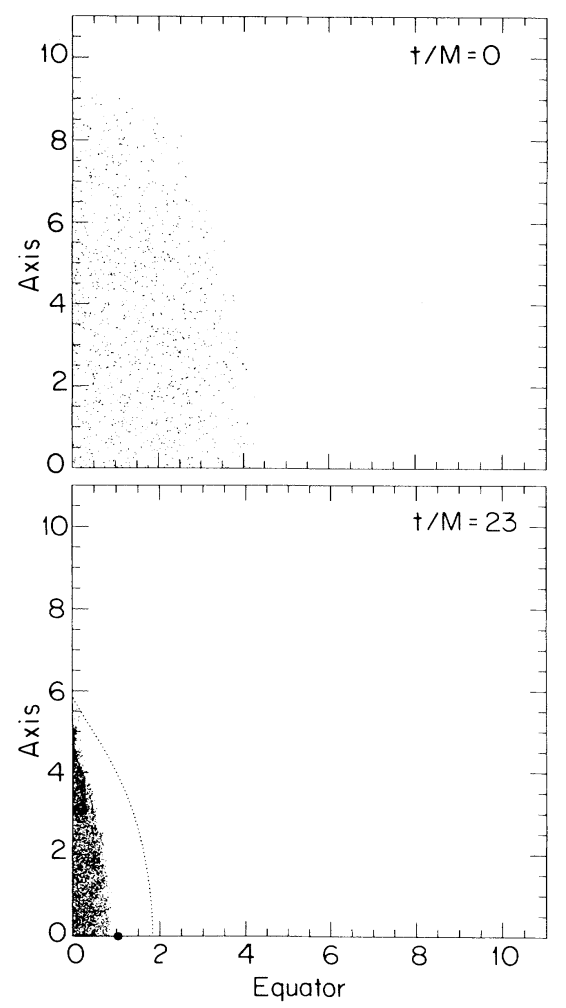

FIG. 2. Snapshots of the particle positions at initial and final times for prolate collapse with the same initial eccentricity as Fig. 1 but with initial semimajor axis equal to $10 M$. The collapse proceeds as in Fig. 1, and terminates with the formation of a spindle singularity on the axis at $t / M=23$. The minimum polar circumference is $\mathcal{C}_{\text {pole }}^{\mathrm{min}} / 4 \pi M=2.8$. There is no apparent horizon, in agreement with the hoop conjecture. This is a good candidate for a naked singularity, which would violate the cosmic censorship hypothesis. 
collapse of nonrotating prolate and oblate spheroids of various initial sizes and eccentricities. The matter particles are instantaneously at rest at $t=0$ and the configurations give exact solutions of the relativistic initialvalue equations. ${ }^{6}$ In the Newtonian limit, these spheroids reduce to homogeneous spheroids. When they are large (size $\gg M$ in all directions), we confirm that their evolution is Newtonian. ${ }^{4,10}$

Figure 1 shows the fate of a typical prolate configuration that collapses from a highly compact and relativistic initial state to a black hole. Note that in isotropic coordinates a Schwarzschild black hole on the initial time slice would have radius $r=0.5 \mathrm{M}$, corresponding to a Schwarzschild radius $r_{S}=2 M$. Figure 2 depicts the outcome of prolate collapse with the same initial eccentricity but from a larger semimajor axis. Here the configuration collapses to a spindle singularity at the pole without the appearance of an apparent horizon. ${ }^{11}$ The spindle consists of a concentration of matter near the axis at $r \approx 5 M$. Figure 3 shows the growth of the Riemann invariant $I$ at $r=6.1 M$ on the axis, just outside the matter. Before the formation of the singularity, the typical size of $I$ at any exterior radius $r$ on the axis is $\sim M^{2} / r^{6} \ll 1$. With the formation of the spindle singularity, the value of $I$ rises without bound in the region near the pole. The maximum value of $I$ determined by our code is limited only by the resolution of the angular grid: The better we resolve the spindle, the larger the value of $I$ we can attain before the singularity causes the code (and spacetime) to break down. Unlike shellcrossing singularities, where $I$ blows up in the matter interior whenever the matter density is momentarily infinite, the singularity also extends outside the matter

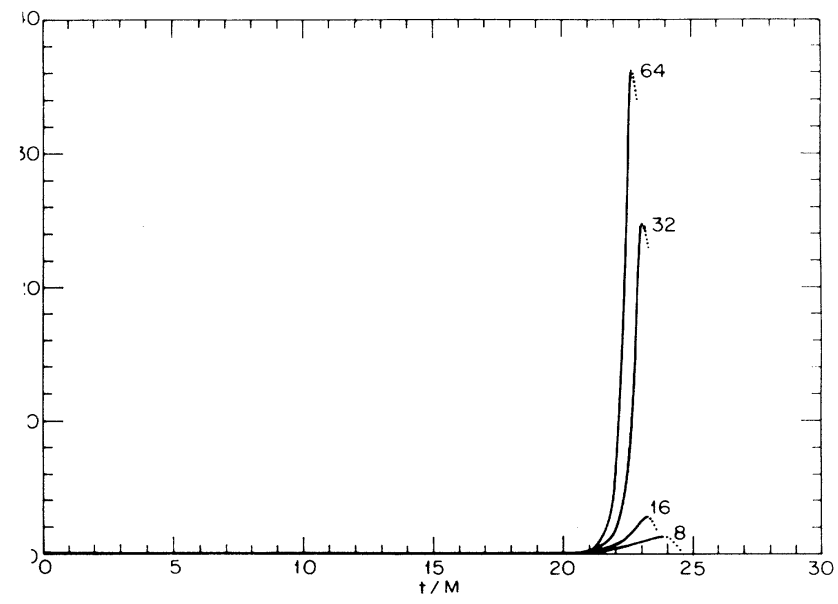

FIG. 3. Growth of the Riemann invariant $I$ (in units of $M^{-4}$ ) vs time for the collapse shown in Fig. 2. The simulation was repeated with various angular grid resolutions. Each curve is labeled by the number of angular zones used. We use dots to show where the singularity has caused the code to become inaccurate. beyond the pole at $r=5.8 M$ (Fig. 4). In fact, the peak value of $I$ occurs in the vacuum at $r \approx 6.1 M$. Here the exterior tidal gravitational field is blowing up, which is not the case for shell crossing. The absence of an apparent horizon suggests that the spindle is a naked singularity.

When our simulation terminates, $I$ along the axis falls to half its peak value at $r \approx 4.5 M$ inside the matter and $r \approx 6.7 M$ outside the matter. The singularity is not a point. Rather it is an extended region which includes the matter spindle, but grows most rapidly in the vacuum exterior above the pole. A $t=$ const slice has a spatial metric

$$
d s^{2}=A^{2} d r^{2}+A^{2} r^{2} d \theta^{2}+B^{2} r^{2} \sin ^{2} \theta d \phi^{2} .
$$

In flat space $A=B=1$. At the termination of the simulation these quantities have a modest maximum value $A \approx B \approx 1.7$, which occurs at the origin. They decrease monotonically outwards, reaching unity at large distances. However, it is their second derivatives that contribute to $I$ and these blow up. While $A$ and $B$ steadily grow with time, $I$ diverges much more rapidly. The behavior is similar to the logarithmic divergence of the metric in the analytic prolate sequence of Ref. 6 . We emphasize that the above characterization of the singularity and the behavior of the metric is dependent on the time slicing and may be different for other choices of time coordinate. In principle, the spindle singularity might first occur at the center rather than the pole with a different time slicing.

The absence of an apparent horizon does not necessarily imply the absence of a global event horizon, although the converse is true. Because such singularities cause our numerical integrations to terminate, we cannot map out a spacetime arbitrarily far into the future, which would be necessary to completely rule out the formation of an event horizon. However, we do not think this is at all likely: For collapse from an initially compact state (Fig. 1), outward null geodesics turn around

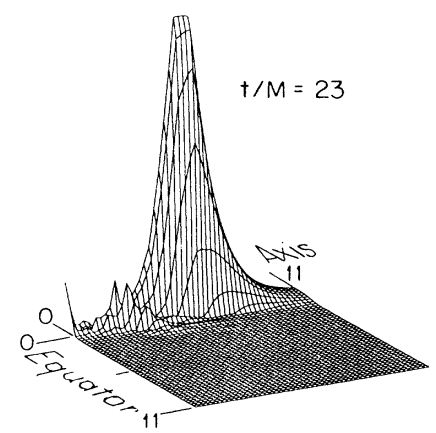

FIG. 4. Profile of $I$ in a meridional plane for the collapse shown in Fig. 2. For the case of 32 angular zones shown here, the peak value of $I$ is $24 / M^{4}$ and occurs on the axis just outside the matter. 
near the singularity. For collapse from large radius, by contrast (Fig. 2), outward null geodesics are still propagating freely away from the vicinity of the singularity up to the time our integrations terminate. It is an interesting question for future research whether any time slicing can be found which will be more effective in snuggling up to the singularity without actually hitting it. ${ }^{12}$ Such a slicing would enable one to confirm that all outward null geodesics propagate to large distances.

Further evidence for the nakedness of the singularity is the similarity of the spindle singularity to the infinite cylinder naked singularity. ${ }^{3,5}$ In both cases the proper length of a given segment of matter along the axis grows slowly, while its proper circumference and surface area shrink to zero much more rapidly. Also, the singularity is an extended region along the axis and not just a point.

We have also followed the collapse of an initially oblate configuration with the same initial eccentricity and semimajor axis as Fig. 2. Following pancaking, it overshoots, becomes prolate, and forms a black hole. At the time our integrations terminate, we find that $\mathcal{C}_{\mathrm{pole}}^{\min }=\mathcal{C}_{\mathrm{eq}}^{\mathrm{min}}$ $=0.85(4 \pi M)$.

All of the above results are consistent with the hoop conjecture. When black holes form, the minimum polar and equatorial circumferences satisfy $\mathcal{C}^{\min } \lesssim 4 \pi M$. Conversely, when naked singularities form, the minimum polar circumference is much bigger than this value. In all cases where an apparent horizon forms, its area satisfies to within numerical accuracy $\mathcal{A} \leq 16 \pi M^{2}$, as required theoretically. ${ }^{13}$ In every case we find that gravitational radiation carries away a negligible fraction $(\ll 1 \%)$ of the total mass energy by the time a black hole or naked singularity forms.

In conclusion, we have presented numerical evidence that the hoop conjecture is a valid criterion for the formation of black holes during nonspherical gravitational collapse. We have also found numerical candidates for the formation of naked singularities from nonsingular initial configurations. These examples are in contrast with any cases of singularities which may arise during spherical collapse. There the exterior spacetime is always the Schwarzschild metric and the Riemann invariant is always exactly $48 M^{2} / r_{S}^{6}$, which is finite outside the matter. In spherical collapse the singularities can thus only occur inside the matter. Here the singularities extend above the pole into the vacuum exterior. These examples suggest that the unqualified cosmic censorship hypothesis cannot be valid.

While the matter treated here has kinetic pressure, it is collisionless, not fluid. We do not regard the collisional properties of the matter as crucial: First, the formation of naked singularities should not depend on the particular details of the fundamental interactions affecting matter at high densities. The gravitational field equations alone should be sufficient to rule out naked singularities, at least in the vacuum exterior, for true cosmic censorship. Second, collisional effects may even ac- celerate the formation of singularities via relativistic "pressure regeneration.", 14

The examples considered here have no angular momentum. The presence of angular momentum could prevent an infinitesimally thin spindle singularity from forming on the axis. Yet we know that a small amount of angular momentum does not prevent the formation of a singularity when a Kerr black hole forms. In any case, it would be disturbing if a collapsing configuration could become arbitrarily close to singular for arbitrarily small angular momentum.

This research was supported in part by National Science Foundation Grants No. AST 87-14475 and No. PHY 90-07834 at Cornell University. Computations were performed on the Cornell National Supercomputer Facility. We thank A. Abrahams, M. Choptuik, C. Evans, and L. S. Finn for helpful discussions.

${ }^{1}$ R. Penrose, Riv. Nuovo Cimento 1 (Numero Special), 252 (1969).

${ }^{2}$ See, e.g., D. S. Goldwirth, A. Ori, and T. Piran, in Frontiers in Numerical Relativity, edited by C. R. Evans, L. S. Finn, and D. W. Hobill (Cambridge Univ. Press, Cambridge, 1989), p. 414, for discussion and references.

${ }^{3}$ K. S. Thorne, in Magic Without Magic: John Archibald Wheeler, edited by J. Klauder (Freeman, San Francisco, 1972), p. 1.

${ }^{4}$ C. C. Lin, L. Mestel, and F. H. Shu, Astrophys. J. 142, 1431 (1965).

${ }^{5}$ C. W. Misner, K. S. Thorne, and J. A. Wheeler, Gravitation (Freeman, San Francisco, 1973), p. 867.

${ }^{6}$ T. Nakamura, S. L. Shapiro, and S. A. Teukolsky, Phys. Rev. D 38, 2972 (1988).

${ }^{7}$ R. Arnowitt, S. Deser, and C. W. Misner, in Gravitation: An Introduction to Current Research, edited by L. Witten (Wiley, New York, 1962), p. 227.

${ }^{8}$ C. R. Evans, Ph.D. thesis, The University of Texas at Austin, 1984 (unpublished).

${ }^{9}$ S. L. Shapiro and S. A. Teukolsky, Astrophys. J. 298, 34 (1985); 298, 58 (1985); 307, 575 (1986); Astrophys. J. (Lett.), 292, L41 (1985).

${ }^{10}$ S. L. Shapiro and S. A. Teukolsky, Astrophys. J. 318, 542 (1987).

"We searched for both a single global horizon centered on the origin as well as a small disjoint horizon around the singularity in each hemisphere.

${ }^{12}$ Maximal slicing apparently does not hold back the formation of prolate spindle singularities. For prolate spheroids the Newtonian potential diverges only logarithmically as the eccentricity $\rightarrow 1$, which may explain why $\alpha$ does not plummet precipitously near a spindle.

${ }^{13}$ See, e.g., D. M. Eardley, Phys. Rev. D 12, 3072 (1975).

${ }^{14}$ See, e.g., Ref. 5, p. 605 . Moreover, there is at least one tentative numerical example [T. Nakamura and H. Sato, Prog. Theor. Phys. 68, 1396 (1982); T. Nakamura, K. Oohara, and Y. Kojima, Prog. Theor. Phys. Suppl. 90, 57 (1987)] of prolate fluid collapse (adiabatic index $\Gamma \rightarrow 2$ ) that appears to be evolving to a singular state without the formation of an apparent horizon. 